\title{
COHEN-MACAULAY EDGE IDEAL WHOSE HEIGHT IS HALF OF THE NUMBER OF VERTICES
}

\author{
MARILENA CRUPI, GIANCARLO RINALDO, AND \\ NAOKI TERAI
}

\begin{abstract}
We consider a class of graphs $G$ such that the height of the edge ideal $I(G)$ is half of the number $\sharp V(G)$ of the vertices. We give Cohen-Macaulay criteria for such graphs.
\end{abstract}

\section{§0. Introduction}

In this article, a graph means a simple graph without loops and multiple edges. Let $G$ be a graph with the vertex set $V(G)=\left\{x_{1}, \ldots, x_{n}\right\}$ and with the edge set $E(G)$. Let $S=K\left[x_{1}, \ldots, x_{n}\right]$ be the polynomial ring in $n$ variables over a field $K$. The edge ideal $I(G)$, associated to $G$, is the ideal of $S$ generated by the set of all square-free monomials $x_{i} x_{j}$ so that $x_{i}$ is adjacent to $x_{j}$. For this ideal, the following theorem is known.

TheOREm 0.1 (see [5]). Suppose that $G$ is an unmixed graph without isolated vertices. Then we have 2 height $I(G) \geq \sharp V(G)$.

In this article, we treat the class of graphs for which the above equality holds; that is, we consider an unmixed graph without isolated vertex with 2 height $I(G)=\sharp V(G)$. Such a class of graphs is rich, because it includes all the unmixed bipartite graphs and all the grafted graphs. Herzog and Hibi [8] gave beautiful theorems on Cohen-Macaulay edge ideals of bipartite graphs. Our purpose in this article is to generalize their results for our class of graphs.

Received May 19, 2009. Revised March 8, 2010. Accepted May 9, 2010.

2000 Mathematics Subject Classification. Primary 05C75; Secondary 05C90, 13H10, $55 \mathrm{U} 10$.

Terai's work partially supported by Gruppo Nazionale per le Strutture Algebriche e Geometriche e loro Applicazioni - Instituto Nazionale di Alta Matematica and by KAKENHI-18540041 and KAKENHI-20540047. 
It is known that a graph $G$ in our class has a perfect matching (see $[6$, Remark 2.2]). We may assume that

$$
V(G)=X \cup Y, \quad X \cap Y=\emptyset,
$$

where $X=\left\{x_{1}, \ldots, x_{n}\right\}$ is a minimal vertex cover of $G$ and where $Y=$ $\left\{y_{1}, \ldots, y_{n}\right\}$ is a maximal independent set of $G$ such that $\left\{x_{1} y_{1}, \ldots, x_{n} y_{n}\right\} \subset$ $E(G)$.

Hence, $\left\{x_{1}-y_{1}, \ldots, x_{n}-y_{n}\right\}$ is a system of parameters of $S / I(G)$. In Sections 3 and 4 , using assumption $\left(^{*}\right)$, we give the following characterization of Cohen-Macaulayness, which is similar to the case of bipartite graphs (see [8, Corollary 3.5]).

THEOREM 0.2. Let $G$ be an unmixed graph with $2 n$ vertices, which are not isolated, and with height $I(G)=n$. Then the following conditions are equivalent.

(1) $G$ is Cohen-Macaulay.

(2) $\Delta(G)$ is strongly connected.

(3) There is a unique perfect matching in $G$.

(4) $G$ is shellable.

Note that it includes equivalence between Cohen-Macaulayness and shellability as in the bipartite graphs (see [3]).

We also have a Cohen-Macaulay criterion which is similar to that of Herzog and Hibi [8, Theorem 3.4].

THEOREM 0.3. Let $G$ be a graph with $2 n$ vertices, which are not isolated, and with height $I(G)=n$. We assume conditions (*) and

$$
x_{i} y_{j} \in E(G) \text { implies } i \leq j \text {. }
$$

Then the following conditions are equivalent.

(1) $G$ is Cohen-Macaulay.

(2) $G$ is unmixed.

(3) The following conditions hold:

(i) if $z_{i} x_{j}, y_{j} x_{k} \in E(G)$, then $z_{i} x_{k} \in E(G)$ for distinct $i, j, k$ and for $z_{i} \in\left\{x_{i}, y_{i}\right\}$

(ii) if $x_{i} y_{j} \in E(G)$, then $x_{i} x_{j} \notin E(G)$.

Although in Herzog and Hibi [8] Alexander duality plays an important role in their proof, we give a direct and elementary proof without it. The 
Herzog-Hibi criterion for bipartite graphs is discussed by other authors in the literature that give alternative proofs for it (see [7], [12]).

In Section 5, we introduce a new class of graphs which we call $B$-grafted graphs. They are a generalization of grafted graphs introduced by Faridi [4]. If $G$ is an unmixed B-grafted graph, then we have 2 height $I(G)=\sharp V(G)$. Hence, applying our main result, we show the following.

TheOrem 0.4. The B-grafted graph $G\left(H_{0} ; B_{1}, \ldots, B_{p}\right)$ is Cohen-Macaulay (resp., unmixed) if and only if every bipartite graph $B_{i}$ is Cohen-Macaulay (resp., unmixed) for $i=1, \ldots, p$.

See Sections 1 and 5 for undefined concepts and notation.

\section{$\S 1$. Preliminaries}

In this section, we recall some concepts and a notation on graphs and on simplicial complexes that we use in the article.

Let $G$ be a graph with the vertex set $V(G)=\left\{x_{1}, \ldots, x_{n}\right\}$ and with the edge set $E(G)$. The induced subgraph $\left.G\right|_{W}$ by $W \subset V(G)$ is defined by

$$
\left.G\right|_{W}=(W,\{e \in E(G) ; e \subset W\})
$$

For $W \subset V(G)$, we denote $\left.G\right|_{V(G) \backslash W}$ by $G-W$. For $F \subset E(G)$, we denote $(V(G), E(G) \backslash F)$ by $G-F$. For a family $F$ of two-element subsets of $V(G)$, we denote $(V(G), E(G) \cup F)$ by $G+F$.

A subset $C \subset V(G)$ is a vertex cover of $G$ if every edge of $G$ is incident with at least one vertex in $C$. A vertex cover $C$ of $G$ is called minimal if there is no proper subset of $C$ which is a vertex cover of $G$. A subset $A$ of $V(G)$ is called an independent set of $G$ if no two vertices of $A$ are adjacent. An independent set $A$ of $G$ is maximal if there exists no independent set which properly includes $A$. Observe that $C$ is a minimal vertex cover of $G$ if and only if $V(G) \backslash C$ is a maximal independent set of $G$. And also note that height $I(G)$ is equal to the smallest number $\sharp C$ of vertices among all the minimal vertex covers $C$ of $G$. A graph $G$ is called unmixed if all the minimal vertex covers of $G$ have the same number of elements. A graph $G$ is called Cohen-Macaulay if $S / I(G)$ is a Cohen-Macaulay ring, where $S=K\left[x_{1}, \ldots, x_{n}\right]$ is a polynomial ring for a field $K$.

Finally, a subgraph $H$ of a graph $G$ with $V(G)=V(H)$ is called a perfect matching if every connected component of $H$ is a 2-complete graph.

See [2] and [13] for detailed information on this subject. 
Set $V=\left\{x_{1}, \ldots, x_{n}\right\}$. A simplicial complex $\Delta$ on the vertex set $V$ is a collection of subsets of $V$ such that (i) $\left\{x_{i}\right\} \in \Delta$ for all $x_{i} \in V$ and (ii) $F \in \Delta$ and $G \subseteq F$ imply $G \in \Delta$. An element $F \in \Delta$ is called a face of $\Delta$. For $F \subset V$, we define the dimension of $F$ by $\operatorname{dim} F=\sharp F-1$, where $\sharp F$ is the cardinality of the set $F$. A maximal face of $\Delta$ with respect to inclusion is called a facet of $\Delta$. If all facets of $\Delta$ have the same dimension, then $\Delta$ is called pure.

A pure simplicial complex $\Delta$ is called shellable if the facets of $\Delta$ can be given a linear order $F_{1}, \ldots, F_{m}$ such that for all $1 \leq j<i \leq m$, there exist some $v \in F_{i} \backslash F_{j}$ and some $k \in\{1, \ldots, i-1\}$ with $F_{i} \backslash F_{k}=\{v\}$.

Moreover, a pure simplicial complex $\Delta$ is strongly connected if for every two facets $F$ and $G$ of $\Delta$ there is a sequence of facets $F=F_{0}, F_{1}, \ldots, F_{m}=G$ such that $\operatorname{dim}\left(F_{i} \cap F_{i+1}\right)=\operatorname{dim} \Delta-1$ for each $i=0, \ldots, m-1$.

If $G$ is a graph, we define the complementary simplicial complex of $G$ by

$$
\Delta(G)=\{A \subseteq V(G): A \text { is an independent set in } G\} .
$$

Observe that $\Delta(G)$ is the Stanley-Reisner simplicial complex of $I(G)$.

A graph $G$ is called shellable if $\Delta(G)$ is a shellable simplicial complex.

\section{$\S 2$. Unmixedness}

In this section, we survey unmixed graphs whose edge ideals have the height that is half of the number of vertices.

LEMMA 2.1. Let $G$ be an unmixed graph with nonisolated $2 n$ vertices and with height $I(G)=n$. Then $G$ has a perfect matching.

This fact is written in [6, Remark 2.2]. By the lemma for an unmixed graph $G$ with $2 n$ vertices, which are not isolated, and with height $I(G)=n$, we may assume that

$$
V(G)=X \cup Y, \quad X \cap Y=\emptyset,
$$

where $X=\left\{x_{1}, \ldots, x_{n}\right\}$ is a minimal vertex cover of $G$ and where $Y=$ $\left\{y_{1}, \ldots, y_{n}\right\}$ is a maximal independent set of $G$ such that $\left\{x_{1} y_{1}, \ldots, x_{n} y_{n}\right\} \subset$ $E(G)$.

For the remainder of this article, set $S=K\left[x_{1}, \ldots, x_{n}, y_{1}, \ldots, y_{n}\right]$ for a field $K$, and $I(G)$ is an ideal of $S$. By Lemma 2.1, we have the following ring-theoretic properties of $S / I(G)$.

COROLlary 2.2. Let $G$ be an unmixed graph with $2 n$ vertices, which are not isolated, and with height $I(G)=n$. We assume condition (*). Then, 
(i) each minimal prime ideal of $I(G)$ is of the form

$$
\left(x_{i_{1}}, \ldots, x_{i_{k}}, y_{i_{k+1}}, \ldots, y_{i_{n}}\right) \text {, }
$$

where $\left\{i_{1}, \ldots, i_{n}\right\}=\{1, \ldots, n\}$;

(ii) $\left\{x_{1}-y_{1}, \ldots, x_{n}-y_{n}\right\}$ is a system of parameters of $S / I(G)$.

For later use we give a characterization of the unmixedness for our graphs, that is, a more detailed description, but a special case of a more general result (see [10, Theorem 2.9] and see [14, Theorem 1.1] for the bipartite case).

Proposition 2.3. Let $G$ be a graph with $2 n$ vertices, which are not isolated, and with height $I(G)=n$. We assume condition $\left(^{*}\right)$. Then $G$ is unmixed if and only if the following conditions hold.

(i) If $z_{i} x_{j}, y_{j} x_{k} \in E(G)$, then $z_{i} x_{k} \in E(G)$ for distinct $i, j, k$ and for $z_{i} \in\left\{x_{i}, y_{i}\right\}$.

(ii) If $x_{i} y_{j} \in E(G)$, then $x_{i} x_{j} \notin E(G)$.

\section{$\S 3$. Cohen-Macaulayness}

In this section, we give combinatorial characterizations of Cohen-Macaulay graphs whose edge ideals have the height that is half of the number of vertices.

First, we introduce an operator that allows us to construct a new graph. Let $G$ be a graph with $2 n$ vertices, which are not isolated, and with height $I(G)=$ $n$. We assume condition $(*)$.

For any $i \in[n]:=\{1, \ldots, n\}$, set

$$
E_{i}:=\left\{k \in[n]: x_{k} y_{i} \in E(G)\right\} \backslash\{i\},
$$

and define the graph $O_{i}(G)$ by

$$
O_{i}(G):=G-\left\{x_{k} y_{i}: k \in E_{i}\right\}+\left\{x_{k} x_{i}: k \in E_{i}\right\} .
$$

Then, for every nonempty subset $T:=\left\{i_{1}, \ldots, i_{\ell}\right\}$ of the set $[n]$, we define

$$
O_{T}(G)=O_{i_{1}} O_{i_{2}} \cdots O_{i_{\ell}}(G) .
$$

Moreover, if $T=\emptyset$, then we set $O_{T}(G)=G$. Note that $O_{T}(G)$ is a graph with $2 n$ vertices, which are not isolated, and with height $I(G)=n$, satisfying condition $(*)$. 
EXAMPLE 3.1. Let $T=\{2,3\}$; then

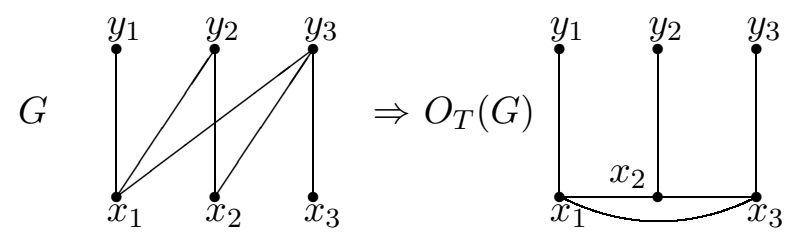

The next proposition shows that the Cohen-Macaulayness of $G$ can be checked by the unmixedness of all the deformations $O_{T}(G)$ of $G$.

Proposition 3.2. Let $G$ be an unmixed graph with $2 n$ vertices, which are not isolated, and with height $I(G)=n$. We assume condition $\left(^{*}\right)$. Then the following conditions are equivalent.

(1) $G$ is Cohen-Macaulay.

(2) $O_{T}(G)$ is Cohen-Macaulay for every subset $T$ of $[n]$.

(3) $O_{T}(G)$ is unmixed for every subset $T$ of $[n]$.

Proof. Set $S=K\left[x_{1}, \ldots, x_{n}, y_{1}, \ldots, y_{n}\right]$, set $S_{k}=K\left[x_{1}, \ldots, x_{n}, y_{k+1}, \ldots\right.$, $\left.y_{n}\right]$, and set $G_{k}=\left.O_{T_{k}}(G)\right|_{X \cup\left\{y_{k+1}, \ldots, y_{n}\right\}}$.

$(1) \Longrightarrow(2)$. By relabeling, we may assume that $T=[k]$. Let $G$ be a Cohen-Macaulay graph. Then

$$
S /\left(I(G)+\left(x_{1}-y_{1}, \ldots, x_{k}-y_{k}\right)\right) \simeq S_{k} /\left(I\left(G_{k}\right)+\left(x_{1}^{2}, \ldots, x_{k}^{2}\right)\right)
$$

is Cohen-Macaulay. Since the polarization preserves Cohen-Macaulayness,

$$
S /\left(I\left(G_{k}\right)+\left(x_{1}^{2}, \ldots, x_{k}^{2}\right)\right)^{\mathrm{pol}}=S /\left(I\left(G_{k}\right)+\left(x_{1} y_{1}, \ldots, x_{k} y_{k}\right)\right)=S / I\left(O_{T}(G)\right)
$$

is Cohen-Macaulay, where $\left(x_{1}^{2}, \ldots, x_{k}^{2}\right)^{\text {pol }}$ stands for the polarization of $\left(x_{1}^{2}\right.$, $\left.\ldots, x_{k}^{2}\right)$. See [11] for basic properties of polarization.

$(2) \Longrightarrow(3)$. Every Cohen-Macaulay ideal is unmixed (see [1]).

$(3) \Longrightarrow(1)$. Suppose that $G$ is not Cohen-Macaulay. We want to prove that there exists a subset $T \subset[n]$ such that $O_{T}(G)$ is not unmixed. Since $G$ is not Cohen-Macaulay, the sequence $\left\{x_{i}-y_{i}: 1 \leq i \leq n\right\}$ is not a regular sequence of $S / I(G)$. Hence, there exists $k \geq 1$ such that $\left\{x_{i}-y_{i}: i \in[k-1]\right\}$ is a regular sequence of $S / I(G)$ and $x_{k}-y_{k}$ is not regular on the ring $R:=$ $S_{k-1} /\left(I\left(G_{k-1}\right)+\left(x_{1}^{2}, \ldots, x_{k-1}^{2}\right)\right) \simeq S /\left(I(G)+\left(x_{1}-y_{1}, \ldots, x_{k-1}-y_{k-1}\right)\right)$. Set $J=I\left(G_{k-1}\right)+\left(x_{1}^{2}, \ldots, x_{k-1}^{2}\right)$. Since $x_{k}-y_{k}$ is not regular on $R$, then

$$
x_{k}-y_{k} \in \bigcup_{P \in \operatorname{Ass} R} P \text {, }
$$


and there exists an associated prime ideal $\widetilde{P}$ of $J$ such that $x_{k}-y_{k} \in \widetilde{P}$. Since $x_{k} \in \widetilde{P}$ or $y_{k} \in \widetilde{P}$, we have $x_{k}, y_{k} \in \widetilde{P}$. Hence, height $\widetilde{P}>n$. Hence, $R$ is not unmixed. Therefore, $S /\left(I\left(G_{k-1}\right)+\left(x_{1}^{2}, \ldots, x_{k-1}^{2}\right)\right)^{\text {pol }} \simeq S / I\left(O_{T_{k-1}}(G)\right)$ is not unmixed.

For distinct $i_{1}, i_{2}, \ldots, i_{r} \in[n]$, we denote by $C_{i_{1} i_{2} \cdots i_{r}}$ the cycle $C$ with

$$
V(C)=\left\{x_{i_{1}}, y_{i_{1}}, x_{i_{2}}, \ldots, x_{i_{r}}, y_{i_{r}}\right\}
$$

and

$$
E(C)=\left\{x_{i_{1}} y_{i_{1}}, y_{i_{1}} x_{i_{2}}, x_{i_{2}} y_{i_{2}}, \ldots, y_{i_{r}} x_{i_{r}}, y_{i_{r}} x_{i_{1}}\right\} .
$$

Proposition 3.3. Let $G$ be an unmixed graph with $2 n$ vertices, which are not isolated, and with height $I(G)=n$. We assume condition (*). Then the following conditions are equivalent.

(1) The subset $\left\{x_{1} y_{1}, x_{2} y_{2}, \ldots, x_{n} y_{n}\right\}$ of $E(G)$ is a unique perfect matching in $G$.

(2) The cycle $C_{i j}$ is not a subgraph of $G$ for any $i<j$.

(3) For any $r \geq 2$, the cycle $C_{i_{1} i_{2} \cdots i_{r}}$ is not a subgraph of $G$ for any subset $\left\{i_{1}, i_{2}, \ldots, i_{r}\right\} \subset[n]$ of cardinality $r$.

Proof. (1) $\Longrightarrow(2)$. Suppose that $C_{i j}$ is a subgraph of $G$. Then we have two perfect matchings in $G$ :

$$
\begin{gathered}
\left\{x_{1} y_{1}, x_{2} y_{2}, \ldots, x_{n} y_{n}\right\}, \\
\left\{x_{1} y_{1}, x_{2} y_{2}, \ldots, x_{i-1} y_{i-1}, x_{i} y_{j}, x_{j} y_{i}, x_{i+1} y_{i+1}, \ldots, x_{n} y_{n}\right\} .
\end{gathered}
$$

$(2) \Longrightarrow(3)$. We proceed by induction on $r$.

For $r=2$ there is nothing to prove. Assume that $r>2$, and suppose that $C_{i_{1} i_{2} \cdots i_{r}}$ is a subgraph of $G$. Since $y_{i_{r-1}} x_{i_{r}}, y_{i_{r}} x_{i_{1}} \in E(G)$, we have $y_{i_{r-1}} x_{i_{1}} \in E(G)$ by Proposition 2.3. Hence, $C_{i_{1} i_{2} \cdots i_{r-1}}$ is a subgraph of $G$, which is a contradiction with the inductive hypothesis.

$(3) \Longrightarrow(1)$. Suppose that there exists another perfect matching:

$$
\left\{x_{1} y_{i_{1}}, x_{2} y_{i_{2}}, \ldots, x_{n} y_{i_{n}}\right\} \subset E(G) .
$$

Then we define a permutation $\sigma$ by

$$
\sigma=\left(\begin{array}{cccc}
1 & 2 & \cdots & n \\
i_{1} & i_{2} & \cdots & i_{n}
\end{array}\right) .
$$

Then $\sigma$ can be decomposed as $\sigma=\prod \sigma_{i}$, where each $\sigma_{i}$ is a cycle of $\sigma$. Since $\sigma$ is not an identity permutation, for some $i$ the cycle $\sigma_{i}$ is of the form $\left(j_{1} j_{2} \cdots j_{r}\right)$ with $r \geq 2$. Then we have that $C_{j_{r} j_{r-1} \cdots j_{1}}$ is a subgraph of $G$. 
Now we give characterizations of Cohen-Macaulayness, which is analogous to the corresponding result for bipartite graphs (see [8, Corollary 3.5]).

THEOREM 3.4. Let $G$ be an unmixed graph with $2 n$ vertices, which are not isolated, and with height $I(G)=n$ satisfying condition (*). Then the following conditions are equivalent.

(1) $G$ is Cohen-Macaulay.

(2) $\Delta(G)$ is strongly connected.

(3) The cycle $C_{i j}$ is not a subgraph of $G$ for any $i<j$.

Proof. (1) $\Longrightarrow(2)$. This is well known.

$(2) \Longrightarrow(3)$. Assume that $C_{i j}$ is a subgraph of $G$ for some $i<j$. Let $F$ be a facet of $\Delta(G)$ such that $x_{i} \in F$. Since $x_{i} y_{j} \in E(G)$, we have $y_{j} \notin F$, and by the unmixedness of $G$ it follows that $x_{j} \in F$. Hence, $\left\{x_{i}, x_{j}\right\} \subset F$. Let $F^{\prime}$ be a facet of $\Delta(G)$ such that $\left\{y_{i}, y_{j}\right\} \subset F^{\prime}$.

We show that there does not exist a chain of facets of $\Delta(G)$ such that

$$
F=F_{0}, F_{1}, \ldots, F_{m}=F^{\prime}, \quad \text { with } \sharp\left(F_{i} \cap F_{i+1}\right)=n-1 \text { for } i=1, \ldots, m-1 \text {. }
$$

Every facet $H \in \Delta(G)$ is one of the following forms:

$$
H=\left\{z_{1}, \ldots, z_{i-1}, x_{i}, z_{i+1}, \ldots, z_{j-1}, x_{j}, z_{j+1}, \ldots, z_{n}\right\}
$$

or

$$
H=\left\{z_{1}, \ldots, z_{i-1}, y_{i}, z_{i+1}, \ldots, z_{j-1}, y_{j}, z_{j+1}, \ldots, z_{n}\right\},
$$

where $z_{k} \in\left\{x_{k}, y_{k}\right\}$, since $\left\{x_{i} y_{i}, x_{j} y_{j}, x_{i} y_{j}, x_{j} y_{i}\right\} \subset E(G)$. Hence, it is impossible to find such a chain. Hence, $\Delta(G)$ is not strongly connected.

$(3) \Longrightarrow(1)$. In order to prove the statement by Proposition 3.2 , it is sufficient to verify that $O_{T}(G)$ is unmixed for every subset $T$ of $[n]$. In contrast, suppose that there exists $T \subset[n]$ such that $G^{\prime}:=O_{T}(G)$ is not unmixed. By Proposition 2.3, one of the following cases occurs:

(i.a) there exist distinct $i, j, k \in[n]$ such that $x_{i} x_{j}, y_{j} x_{k} \in E\left(G^{\prime}\right)$ but $x_{i} x_{k} \notin$ $E\left(G^{\prime}\right)$;

(i.b) there exist distinct $i, j, k \in[n]$ such that $y_{i} x_{j}, y_{j} x_{k} \in E\left(G^{\prime}\right)$ but $y_{i} x_{k} \notin$ $E\left(G^{\prime}\right)$

(ii) there exist distinct $i, j \in[n]$ such that $x_{i} y_{j}, x_{i} x_{j} \in E\left(G^{\prime}\right)$.

In case (i.a), since $j \notin T$, we have $y_{j} x_{k} \in E(G)$. Moreover, since $j \notin T$, $x_{i} x_{j} \in E\left(G^{\prime}\right)$ implies that

(i.aa) $x_{i} x_{j} \in E(G)$ 
or

(i.ab) $y_{i} x_{j} \in E(G)$ and $i \in T$.

In subcase (i.aa), we have $x_{i} x_{k} \in E(G)$ by Proposition 2.3. Hence, $x_{i} x_{k} \in$ $E\left(G^{\prime}\right)$. This contradicts $x_{i} x_{k} \notin E\left(G^{\prime}\right)$.

In subcase (i.ab), we have $y_{i} x_{k} \in E(G)$ by Proposition 2.3 with $i \in T$. Hence, $x_{i} x_{k} \in E\left(G^{\prime}\right)$. This contradicts $x_{i} x_{k} \notin E\left(G^{\prime}\right)$.

In case (i.b), $y_{i} x_{j}, y_{j} x_{k} \in E\left(G^{\prime}\right)$ implies that $i, j \notin T$. Hence, $y_{i} x_{j}, y_{j} x_{k} \in$ $E(G)$. Then $y_{i} x_{k} \in E(G)$ by Proposition 2.3. Hence, $y_{i} x_{k} \in E\left(G^{\prime}\right)$. This contradicts $y_{i} x_{k} \notin E\left(G^{\prime}\right)$.

In case (ii), $x_{i} y_{j} \in E\left(G^{\prime}\right)$ implies that $j \notin T$. Hence, $x_{i} y_{j} \in E(G)$. Moreover, $x_{i} x_{j} \in E\left(G^{\prime}\right)$ implies that

(ii.a) $y_{i} x_{j} \in E(G)$ and $i \in T$

or

(ii.b) $x_{i} x_{j} \in E(G)$.

In subcase (ii.a), we have $y_{i} x_{j}, y_{j} x_{i} \in E(G)$. This contradicts the assumption that $C_{i j}$ is not a subgraph of $G$.

In subcase (ii.b), we have $x_{i} x_{j}, x_{i} y_{j} \in E(G)$. Hence, $G$ is not unmixed by Proposition 2.3. This contradicts the assumption that $G$ is unmixed.

The next lemma is crucial for giving another criterion for the CohenMacaulayness of our graphs.

LEMMA 3.5. Let $G$ be an unmixed graph with $2 n$ vertices, which are not isolated, and with height $I(G)=n$. We assume condition (*).

If $G$ is a Cohen-Macaulay graph, then there exists a suitable simultaneous change of labeling on both $\left\{x_{i}\right\}$ and $\left\{y_{i}\right\}$ (i.e., we relabel $\left(x_{i_{1}}, \ldots, x_{i_{n}}\right)$ and $\left(y_{i_{1}}, \ldots, y_{i_{n}}\right)$ as $\left(x_{1}, \ldots, x_{n}\right)$ and $\left(y_{1}, \ldots, y_{n}\right)$ at the same time), such that $x_{i} y_{j} \in E(G)$ implies that $i \leq j$.

Proof. We can define a partial order $\preceq$ on $X$ by

$$
x_{i} \preceq x_{j} \quad \text { if and only if } x_{i} y_{j} \in E(G) .
$$

In fact, the reflexivity holds by condition $(*)$, the transitivity holds by unmixedness of $G$ (see Proposition 2.3(i)), and the antisymmetry holds since $G$ contains no cycle $C_{i j}$ for any $i<j$. Take a linear extension of $\preceq$, which we call $\preceq^{\prime}$. By the linear order $\preceq^{\prime}$, we have $x_{i_{1}} \preceq^{\prime} \cdots \preceq^{\prime} x_{i_{n}}$. We relabel them as $x_{1} \preceq^{\prime} \ldots \preceq^{\prime} x_{n}$. At the same time, we relabel $y_{i_{1}}, \ldots, y_{i_{n}}$ as $y_{1}, \ldots, y_{n}$. Then if $x_{i} y_{j} \in E(G), x_{i} \preceq^{\prime} x_{j}$. Hence, $i \leq j$. 
Hence, for a Cohen-Macaulay graph $G$ with $2 n$ vertices, which are not isolated, and with height $I(G)=n$ satisfying condition $\left(^{*}\right)$, we may assume that

$$
x_{i} y_{j} \in E(G) \text { implies } i \leq j .
$$

Now we state another Cohen-Macaulay criterion on our graphs, which is a generalization of Herzog and Hibi ([8, Theorem 3.4]).

THEOREM 3.6. Let $G$ be a graph with $2 n$ vertices, which are not isolated, and with height $I(G)=n$. We assume conditions (*) and (**). Then the following conditions are equivalent.

(1) $G$ is Cohen-Macaulay.

(2) $G$ is unmixed.

(3) The following conditions hold:

(i) if $z_{i} x_{j}, y_{j} x_{k} \in E(G)$, then $z_{i} x_{k} \in E(G)$ for distinct $i, j, k$ and for $z_{i} \in\left\{x_{i}, y_{i}\right\}$

(ii) if $x_{i} y_{j} \in E(G)$, then $x_{i} x_{j} \notin E(G)$.

Proof. (1) $\Longrightarrow(2)$. This is well known.

$(2) \Longrightarrow(1)$. This follows from Theorem 3.4, since we assume condition $(* *)$.

$(2) \Longleftrightarrow(3)$. This follows from Proposition 2.3.

We remark that the equivalence between (1) and (2) in Theorem 3.6 is a special case of [9, Theorem 4.3].

As an easy consequence of the previous results, we obtain the upper bound for the minimal number $\mu(I(G))$ of generators of $I(G)$, as follows.

Corollary 3.7. Let $G$ be a graph with $2 n$ vertices, which are not isolated, and with height $I(G)=n$. Then we have the following.

(i) If $G$ is unmixed, then $\mu(I(G)) \leq n^{2}$.

(ii) If $G$ is Cohen-Macaulay, then $\mu(I(G)) \leq(n(n+1)) / 2$.

Proof. The statements are consequences of the criteria for unmixedness and for Cohen-Macaulayness given by Proposition 2.3 and Theorem 3.6.

\section{§4. Shellability and Cohen-Macaulay type}

In this section, if $G$ is a graph such that $\sharp V(G)=2 n$ and height $I(G)=n$, we show the equivalence between the Cohen-Macaulayness and shellability 
of $G$. We also express the Cohen-Macaulay type of $S / I(G)$ in a combinatorial way.

THEOREM 4.1. Let $G$ be an unmixed graph with $2 n$ vertices, which are not isolated, and with height $I(G)=n$. Then $G$ is Cohen-Macaulay if and only if $G$ is shellable.

Here we give a proof only of the following lemma. The rest of the proof is almost identical to the proof of [3, Theorem 2.9].

Lemma 4.2. Let $G$ be a Cohen-Macaulay graph with $2 n$ vertices, which are not isolated, and with height $I(G)=n$. Then there exists a vertex $v \in V(G)$ such that $\operatorname{deg}(v)=1$.

Proof. Since $G$ is Cohen-Macaulay, it is unmixed. By Lemma 2.1, $G$ has a perfect matching. Then we may assume condition $\left(^{*}\right)$. Suppose that each $v \in V(G)$ has at least degree 2 . Let $i_{1}, i_{2}, \ldots$ be a sequence such that $y_{i_{1}} x_{i_{2}}, y_{i_{2}} x_{i_{3}}, \ldots \in E(G)$ with $i_{j} \neq i_{j+1}$. Since the cardinality of $Y$ is finite, there must exist integers $s<t$ such that $i_{t}=i_{s}$. We may assume that $i_{s}, i_{s+1}, \ldots, i_{t-1}$ are distinct. This induces that the cycle $C_{i_{s} i_{s+1} \cdots i_{t-1}}$ is a subgraph of $G$. Therefore, $G$ is not Cohen-Macaulay by Proposition 3.3 and Theorem 3.4 .

Now we express the Cohen-Macaulay type of a graph belonging to our class, imitating the bipartite case (see [13, pp. 184-185]).

Lemma 4.3. Let $G$ be a Cohen-Macaulay graph with $2 n$ vertices, which are not isolated, and with height $I(G)=n$. We assume condition (*). Then

$$
\operatorname{Soc}\left(K\left[x_{1}, \ldots, x_{n}\right] /\left(I\left(\left.O_{[n]}(G)\right|_{X}\right)+\left(x_{1}^{2}, \ldots, x_{n}^{2}\right)\right)\right)
$$

is generated by all the monomials $x_{i_{1}} \cdots x_{i_{r}}$ such that $\left\{x_{i_{1}}, \ldots, x_{i_{r}}\right\}$ is a maximal independent set of $\left.O_{[n]}(G)\right|_{X}$.

Proof. The ring $A:=K\left[x_{1}, \ldots, x_{n}\right] /\left(I\left(\left.O_{[n]}(G)\right|_{X}\right)+\left(x_{1}^{2}, \ldots, x_{n}^{2}\right)\right)$ is spanned as a $K$-vector space by the image of 1 and the images of the square-free monomials

$$
x_{i_{1}} \cdots x_{i_{r}}, \quad 1 \leq i_{1}<i_{2}<\cdots<i_{r} \leq n
$$

such that $x_{i_{j}} x_{i_{k}} \notin E\left(\left.O_{[n]}(G)\right|_{X}\right)$, for $j \neq k$; that is, $\left\{x_{i_{1}}, \ldots, x_{i_{r}}\right\}$ is an independent set of $\left.O_{[n]}(G)\right|_{X}$. Since $A$ is an Artinian positively graded algebra, 
Soc $A=\left(0:_{A} A_{+}\right)$is generated by the images of the square-free monomials of form (4.1) such that $\left\{x_{i_{1}}, \ldots, x_{i_{r}}\right\}$ is a maximal independent set of $\left.O_{[n]}(G)\right|_{X}$.

Corollary 4.4. Let $G$ be a Cohen-Macaulay graph with $2 n$ vertices, which are not isolated, and with height $I(G)=n$. We assume condition (*). Then we have the following.

(i) type $S / I(G)=\sharp \Upsilon\left(\left.O_{[n]}(G)\right|_{X}\right)$, where $\Upsilon\left(\left.O_{[n]}(G)\right|_{X}\right)$ is the family of all minimal vertex covers of $\left.O_{[n]}(G)\right|_{X}$. In particular, type $S / I(G)$ is independent from the base field $K$.

(ii) $G$ is level if and only if $\left.O_{[n]}(G)\right|_{X}$ is unmixed. In particular, the levelness of $G$ is independent from the base field $K$.

Proof. Set $S=K\left[x_{1}, \ldots, x_{n}, y_{1}, \ldots, y_{n}\right]$, and set $S_{n}=K\left[x_{1}, \ldots, x_{n}\right]$.

(i) Since $G$ is Cohen-Macaulay and since $\left\{x_{1}-y_{1}, \ldots, x_{n}-y_{n}\right\}$ is a regular sequence, we have

$$
\text { type } \begin{aligned}
S / I(G) & =\operatorname{dim}_{K} \operatorname{Soc} S /\left(I(G)+\left(x_{1}-y_{1}, \ldots, x_{n}-y_{n}\right)\right) \\
& =\operatorname{dim}_{K} \operatorname{Soc} S_{n} /\left(I\left(\left.O_{[n]}(G)\right|_{X}\right)+\left(x_{1}^{2}, \ldots, x_{n}^{2}\right)\right) \\
& =\sharp \Upsilon\left(\left.O_{[n]}(G)\right|_{X}\right)
\end{aligned}
$$

by Lemma 4.3 .

(ii) When $G$ is Cohen-Macaulay, $G$ is level if and only if

$$
\operatorname{Soc} S /\left(I(G)+\left(x_{1}-y_{1}, \ldots, x_{n}-y_{n}\right)\right)
$$

is equigenerated. By Lemma 4.3, it is equivalent that $\left.O_{[n]}(G)\right|_{X}$ is unmixed. $\square$

The next result generalizes [8, Corollary 3.6].

Corollary 4.5. Let $G$ be a Cohen-Macaulay graph with $2 n$ vertices, which are not isolated, and with height $I(G)=n$. We assume condition (*). Then the following conditions are equivalent.

(1) $G$ is Gorenstein.

(2) $I(G)=\left(x_{1} y_{1}, \ldots, x_{n} y_{n}\right)$.

(3) $G$ is a complete intersection.

Proof. $(1) \Rightarrow(2)$. $G$ is Gorenstein if and only if $S / I(G)$ is Cohen-Macaulay and type $S / I(G)=1$. Since $1=$ type $S / I(G)=\sharp \Upsilon\left(\left.O_{[n]}(G)\right|_{X}\right)$, it follows that $\left.O_{[n]}(G)\right|_{X}$ has a unique minimal vertex cover. Hence, $\left.O_{[n]}(G)\right|_{X}$ is isolated $n$ vertices. Hence, $I(G)=\left(x_{1} y_{1}, \ldots, x_{n} y_{n}\right)$. 
$(2) \Rightarrow(3)$. This is true from its definition.

$(3) \Rightarrow(1)$. See [1].

\section{$\S 5$. B-grafted graph}

In this section, we introduce a new class of graphs $G$ with $\sharp V(G)=2 n$ and with height $I(G)=n$, and we study its Cohen-Macaulayness.

Let $H_{0}$ be a graph with the labeled vertices $1,2, \ldots, p$.

For every $i=1, \ldots, p$, let $B_{i}$ be a bipartite graph with labeled partition $X_{i}$ and $Y_{i}$ such that $\sharp X_{i}=\sharp Y_{i}=n_{i}$. (We do not give a label to each vertex of $B_{i}$, but we distinguish the partition $X_{i}$ and $Y_{i}$.) We assume that $B_{i}$ has no isolated vertex for every $i=1, \ldots, p$. We define the graph

$$
G=G\left(H_{0} ; B_{1}, \ldots, B_{p}\right)
$$

as follows. The vertex set of $G$ is $V(G):=X \cup Y$, where $X=X_{1} \cup \cdots \cup X_{p}$ and $Y=Y_{1} \cup \cdots \cup Y_{p}$. The edge set $E(G)$ of $G$ is defined by $x y \in E(G)$ if and only if either there exist $i, j$ such that $x \in X_{i}, y \in X_{j}$, and $i j \in E\left(H_{0}\right)$ or there exists $i$ such that $x \in X_{i}, y \in Y_{i}$, and $x y \in E\left(B_{i}\right)$. We call such a graph $G$ the $B$-grafted graph. Note that $X$ is a minimal vertex cover of $G$ and that $Y$ is a maximal independent set of $G$. Note also that $\sharp V(G)=2\left(\sum_{i=1}^{p} n_{i}\right)$.

EXAMPLE 5.1. Let $H_{0}$ be a cycle of length 3. By the following bipartite graphs $B_{1}, B_{2}$, and $B_{3}$, we obtain the $B$-grafted graph $G$ :

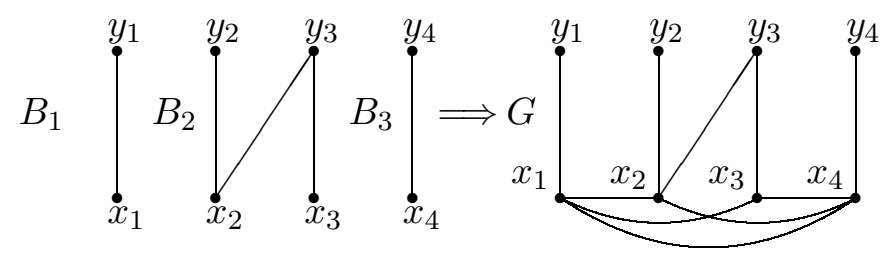

REMARK 5.2. If $B_{i}$ is just a complete graph with two vertices, that is, a complete bipartite graph with $\sharp X_{i}=\sharp Y_{i}=1$ for $i=1, \ldots, p$, then the Bgrafted graph $G$ is called a grafted graph in [4].

Theorem 5.3. The B-grafted graph $G\left(H_{0} ; B_{1}, \ldots, B_{p}\right)$ is Cohen-Macaulay (resp., unmixed) if and only if every bipartite graph $B_{i}$ is Cohen-Macaulay (resp., unmixed) for $i=1, \ldots, p$.

Proof. It is clear from Theorem 3.4 (resp., Proposition 2.3). 
Acknowledgment. The third author gratefully acknowledges the hospitality during his stay at the Department of Mathematics of the University of Messina.

\title{
REFERENCES
}

[1] W. Bruns and J. Herzog, Cohen-Macaulay rings, Cambridge Univ. Press, Cambridge, 1997.

[2] R. Diestel, Graph Theory, 2nd ed., Grad. Texts in Math. 173, Springer, Berlin, 2000.

[3] M. Estrada and R. H. Villarreal, Cohen-Macaulay bipartite graphs, Arch. Math. (Basel) 68 (1997), 124-128.

[4] S. Faridi, "Cohen-Macaulay properties of square-free monomial ideals" in Commutative Algebra, Lect. Notes Pure Appl. Math. 244, Chapman \& Hall, Boca Raton, Fla, 2006, 85-114.

[5] I. Gitler and C. E. Valencia, Bounds for invariants of edge-rings, Comm. Algebra 33 (2005), 1603-1616.

[6] I. Gitler and C. E. Valencia, Bounds for graph invariants, preprint, arXive:math/0510387v2 [math.CO]

[7] H. Haghighi, S. Yassemi, and R. Zaare-Nahandi, Bipartite $S_{2}$ graphs are CohenMacaulay, to appear in Bull. Math. Soc. Sci. Math. Roumanie (N.S.).

[8] J. Herzog and T. Hibi, Distributive lattices, bipartite graphs and Alexander duality, J. Algebraic Combin. 22 (2005), 289-302.

[9] J. Herzog, T. Hibi, and X. Zheng, The monomial ideal of a finite meet-semilattice, Trans. Amer. Math. Soc. 358 (2006), 4119-4134.

[10] S. Morey, E. Reyes, and R. H. Villarreal, Cohen-Macaulay, shellable and unmixed clutters with a perfect matching of König type, J. Pure Appl. Algebra 212 (2008), $1770-1786$.

[11] J. Stückrad and W. Vogel, Buchsbaum Rings and Applications: An Interaction between Algebra, Geometry and Topology, Springer, Berlin, 1986.

[12] A. Van Tuyl and R. H. Villarreal, Shellable graphs and sequentially Cohen-Macaulay bipartite graphs, J. Combin. Theory Ser. A 115 (2008), 779-814.

[13] R. H. Villarreal, Monomial Algebras, Monogr. Textbooks Pure Appl. Math. 238, Marcel Dekker, New York, 2001.

[14] — Unmixed bipartite graphs, Rev. Colombiana Mat. 41 (2007), 393-395.

\author{
Marilena Crupi \\ Dipartimento di Matematica \\ Università di Messina \\ 98166 Messina \\ Italy \\ mcrupi@unime.it
}


Giancarlo Rinaldo

Dipartimento di Matematica

Università di Messina

98166 Messina

Italy

rinaldo@dipmat.unime.it

Naoki Terai

Department of Mathematics

Faculty of Culture and Education

Saga University

Saga 840-8502

Japan

terai@cc.saga-u.ac.jp 